hep-th/9606050

DAMTP 96-54

\title{
A Rational Logarithmic Conformal Field Theory
}

\author{
Matthias R. Gaberdiellin and Horst G. Kausch \\ Department of Applied Mathematics and Theoretical Physics, \\ University of Cambridge, Silver Street, \\ Cambridge CBз 9EW, U.K.
}

10 June 1996

\begin{abstract}
We analyse the fusion of representations of the triplet algebra, the maximally extended symmetry algebra of the Virasoro algebra at $c=-2$. It is shown that there exists a finite number of representations which are closed under fusion. These include all irreducible representations, but also some reducible representations which appear as indecomposable components in fusion products.
\end{abstract}

1. Introduction. Recently a class of conformal field theories whose correlation functions show logarithmic behaviour has attracted some attention. These theories include for example the WZNW model on the supergroup $G L(1,1)$ [1], the $c=-2$ model [2], gravitationally dressed conformal field theories [3] and some critical disordered models [4, [5]. They are believed to be important for the description of certain statistical models, in particular in the theory of (multi)critical polymers [6, 7, 8] and percolation [9]. There have also been suggestions that some of these logarithmic operators might correspond to normalisable zero modes for string backgrounds [10].

These theories are also interesting from a more theoretical point of view. They give rise to novel features in the representation theory of the Virasoro algebra as the appearance of logarithms can be traced back to the property of fusion products [11] to be not completely reducible [2, 12]. So far, all known examples involved an infinite set of representations; in

\footnotetext{
${ }^{*}$ Email: M.R.Gaberdiel@damtp.cam.ac.uk

$\dagger$ Address from 1st September 1996: Department of Physics, Harvard University, Cambridge MA, 02138, USA

${ }_{\ddagger}^{\ddagger}$ Email: H.G.Kausch@damtp.cam.ac.uk

$\S$ Address from 9th September 1996: Department of Mathematics, King's College London, Strand, London WC2R 2LS, U.K.
} 
this letter, we analyse a "rational" logarithmic conformal field theory, i.e. a logarithmic conformal field theory for which a finite set of representations closes under fusion. . Related issues have recently also been discussed by Flohr [7, 13] and in [8, 14, 15].

In [14] we introduced an algorithm to study the fusion products of quasirational representations. We used this algorithm to analyse the fusion of certain representations of the Virasoro $(1, q)$ models (the first of which, the $(1,2)$ model, has $c=-2$ ), and we showed that there exists an infinite set of quasirational representations which is closed under fusion. These representations include irreducible representations, but also some reducible representations which appear as indecomposable components in the fusion products of irreducibles.

It has been known for some time [16], that the Virasoro algebra, the chiral algebra of the Virasoro $(1, q)$ model, can be extended by a triplet of weight $2 q-1$ fields $W^{i}(z)$ to form the triplet algebra. In this letter we shall mainly consider the simplest case, where $q=2$, although much of what we say should also hold in general. The infinitely many quasirational irreducible representations of the Virasoro algebra organise themselves into finitely many irreducible representations of this triplet algebra [8]. Furthermore, as was argued in [17], the triplet algebra has only finitely many irreducible representations. It is then natural to ask whether there exists a finite number of (indecomposable) representations of the triplet algebra which is closed under fusion, and this is indeed what will turn out to be true.

It should be mentioned that Flohr [13 has recently analysed the modular properties of these models. He proposed a partition function for the theory which is constructed from a certain finite set of characters (including the characters of the irreducible representations) which is invariant under the modular group. He then applied the Verlinde formula to obtain "fusion rule coefficients" for corresponding representations, some of which turned out to be negative.

Apart from the fact that the meaning of the negative fusion rule coefficients is rather obscure, it is not clear from his point of view which of his characters actually correspond to representations of the triplet algebra, and if so, which structure they have. In particular, different sets of possible (inequivalent) fusion rules can be found which correspond to different choices for the basis of the characters. It is also not clear in which way the feature of the fusion products to be not completely reducible is taken into account.

In our approach we can deduce the characters corresponding to the finitely many representations which are closed under fusion. It turns out that they are invariant under the modular group, but that a naive application of Verlinde's formula does not make sense. However, there exists some modified procedure (see case III in [13]), by means of which our results can be reproduced from character considerations.

2. The triplet algebra and its irreducible representations. Let us recall the structure of the triplet algebra (at $c=-2$ ). It is generated by the Virasoro modes $L_{n}$, and

\footnotetext{
${ }^{1}$ We should stress that the notion of rationality we use here does not conform with the mathematical literature: there rationality also entails that every representation is completely decomposable into irreducibles, which certainly is not true here.
} 
the modes of a triplet of weight 3 fields $W_{n}^{i}$. The commutation relations are

$$
\begin{aligned}
{\left[L_{m}, L_{n}\right]=} & (m-n) L_{m+n}-\frac{1}{6} m\left(m^{2}-1\right) \delta_{m+n} \\
{\left[L_{m}, W_{n}^{a}\right]=} & (2 m-n) W_{m+n}^{a}, \\
{\left[W_{m}^{a}, W_{n}^{b}\right]=} & g^{a b}\left(2(m-n) \Lambda_{m+n}+\frac{1}{20}(m-n)\left(2 m^{2}+2 n^{2}-m n-8\right) L_{m+n}\right. \\
& \left.\quad-\frac{1}{120} m\left(m^{2}-1\right)\left(m^{2}-4\right) \delta_{m+n}\right) \\
& +f_{c}^{a b}\left(\frac{5}{14}\left(2 m^{2}+2 n^{2}-3 m n-4\right) W_{m+n}^{c}+\frac{12}{5} V_{m+n}^{c}\right)
\end{aligned}
$$

where $\Lambda=: L^{2}:-3 / 10 \partial^{2} L$ and $V^{a}=: L W^{a}:-3 / 14 \partial^{2} W^{a}$ are quasiprimary normal ordered fields. $g^{a b}$ and $f_{c}^{a b}$ are the metric and structure constants of $s u(2)$. In an orthonormal basis we have $g^{a b}=\delta^{a b}, f_{c}^{a b}=i \epsilon^{a b c}$.

The triplet algebra (at $c=-2$ ) is only associative, because certain states in the vacuum representation (which would generically violate associativity) are null. The relevant null vectors are

$$
\begin{aligned}
N^{a}= & \left(2 L_{-3} W_{-3}^{a}-\frac{4}{3} L_{-2} W_{-4}^{a}+W_{-6}^{a}\right)|0\rangle \\
N^{a b}= & W_{-3}^{a} W_{-3}^{b}|0\rangle-g^{a b}\left(\frac{8}{9} L_{-2}^{3}+\frac{19}{36} L_{-3}^{2}+\frac{14}{9} L_{-4} L_{-2}-\frac{16}{9} L_{-6}\right)|0\rangle \\
& -f_{c}^{a b}\left(-2 L_{-2} W_{-4}^{c}+\frac{5}{4} W_{-6}^{c}\right)|0\rangle .
\end{aligned}
$$

We shall only be interested in representations which respect these relations, and for which the spectrum of $L_{0}$ is bounded from below. Then, the zero modes of the null-states have to vanish on the ground states, and this will restrict the allowed representations [18. Evaluating the constraint coming from (2), we find

$$
\left(W_{0}^{a} W_{0}^{b}-g^{a b} \frac{1}{9} L_{0}^{2}\left(8 L_{0}+1\right)-f_{c}^{a b} \frac{1}{5}\left(6 L_{0}-1\right) W_{0}^{c}\right) \psi=0
$$

where $\psi$ is any ground state, while the relation coming from the zero mode of (11) is satisfied identically. Furthermore, the constraint from $W_{1}^{a} N_{-1}^{b c}$, together with (3) implies that $W_{0}^{a}\left(8 L_{0}-3\right)\left(L_{0}-1\right) \psi=0$. Multiplying with $W_{0}^{a}$ and using (3) again, this implies that

$$
0=L_{0}^{2}\left(8 L_{0}+1\right)\left(8 L_{0}-3\right)\left(L_{0}-1\right) \psi .
$$

For irreducible representations, $L_{0}$ has to take a fixed value $h$ on the ground states, and (田) then implies that $h$ has to be either $h=0,-1 / 8,3 / 8$ or $h=1$. It also follows from (3) that

$$
\left[W_{0}^{a}, W_{0}^{b}\right]=\frac{2}{5}(6 h-1) f_{c}^{a b} W_{0}^{c},
$$

which is a rescaled version of the $s u(2)$ algebra. After rescaling, the irreducible representations of these zero modes can then be labelled by $j$ and $m$, where $j(j+1)$ is the 
eigenvalue of the Casimir operator $\sum_{a}\left(W_{0}^{a}\right)^{2}$, and $m$ is the eigenvalue of $W_{0}^{3}$. Because of (官), $W_{0}^{a} W_{0}^{a}=W_{0}^{b} W_{0}^{b}$ on the ground states, and thus $j(j+1)=3 m^{2}$. This can only be satisfied for $j=0,1 / 2$, and this restricts the allowed representations to

- the singlet representations, $j=0$, at $h=0,-1 / 8$;

- the doublet representations, $j=1 / 2$, at $h=1,3 / 8$,

as was remarked in 17.

These four representations have been constructed from a free field realisation in [8]. We shall now briefly discuss their structure, paying particular attention to the special subspace, one of the central concepts in the analysis of fusion [12, 14].

2a. The singlet representation at $h=0$ : This is the vacuum representation, $\mathcal{V}_{0}$. We denote the vacuum state by $\Omega$, and as usual, we have

$$
\begin{aligned}
L_{-n} \Omega & =0, \quad \text { for } n \leq 1, \\
W_{-n}^{a} \Omega & =0, \quad \text { for } n \leq 2 .
\end{aligned}
$$

This implies that the special subspace is trivial, $\mathcal{V}_{0}^{\mathrm{s}}=\langle\Omega\rangle$. Further relations in the vacuum representation are given by (1) and (2).

2b. The singlet representation at $h=-1 / 8$ : We denote the ground state of $\mathcal{V}_{-1 / 8}$ by $\mu$. The constraints coming from the negative modes of the vacuum null vectors (11) and (2) yield $W_{0}^{a} \mu=W_{-1}^{a} \mu=0, L_{-1}^{2} \mu=1 / 2 L_{-2} \mu, L_{-1} W_{-2}^{a} \mu=3 / 4 W_{-3}^{a} \mu$, and

$$
W_{-2}^{a} W_{-2}^{b} \mu=g^{a b}\left(L_{-2}^{2}+\frac{5}{2} L_{-3} L_{-1}-\frac{7}{8} L_{-4}\right) \mu+f_{c}^{a b}\left(2 L_{-2} W_{-2}^{c}+\frac{1}{4} W_{-4}^{c}\right) \mu .
$$

It follows that the special subspace is five-dimensional, and that a basis can be chosen to consist of

$$
\mathcal{V}_{-1 / 8}^{\mathrm{s}}=\left\langle\mu, L_{-1} \mu, W_{-2}^{a} \mu\right\rangle .
$$

2c. The doublet representation at $h=1$ : We denote the two ground states of $\mathcal{V}_{1}$ by $\psi^{ \pm}$. To specify the action of the $W_{0}$ modes on $\psi^{ \pm}$, let us introduce a Cartan-Weyl basis $W^{0}, W^{ \pm}$for $s u(2)$, so that the non-vanishing structure constants are $f_{ \pm}^{0 \pm}=-f_{ \pm}^{ \pm 0}= \pm 1$ and $f^{+-}=-f^{-+}=2$. We also define a metric by $g^{00}=1, g^{+-}=g^{-+}=2$. On the states $\psi^{ \pm}$of the spin $1 / 2$ representation of $s u(2)$, we then define the representation matrices $t^{a}$ by

$$
t^{0} \psi^{ \pm}= \pm \frac{1}{2} \psi^{ \pm}, \quad t^{ \pm} \psi^{ \pm}=0, \quad t^{ \pm} \psi^{\mp}=\psi^{ \pm} .
$$

In this notation, the constraints coming from the null vectors (11) and (2) yield

$$
\begin{aligned}
W_{0}^{a} \boldsymbol{\psi} & =2 t^{a} \boldsymbol{\psi}, & W_{-1}^{a} \boldsymbol{\psi} & =3 L_{-1} t^{a} \boldsymbol{\psi}, \\
W_{-2}^{a} \boldsymbol{\psi} & =4 L_{-2} t^{a} \boldsymbol{\psi}, & L_{-1}^{2} \boldsymbol{\psi} & =2 L_{-2} \boldsymbol{\psi} .
\end{aligned}
$$

It follows that the special subspace is four-dimensional, and that a basis can be chosen to consist of

$$
\mathcal{V}_{1}^{\mathrm{s}}=\left\langle\psi^{ \pm}, L_{-1} \psi^{ \pm}\right\rangle
$$


2d. The doublet representation at $h=3 / 8$ : Finally, we denote the two ground states of $\mathcal{V}_{3 / 8}$ by $\nu^{ \pm}$. The constraints give in this case $W_{0}^{a} \boldsymbol{\nu}=1 / 2 t^{a} \boldsymbol{\nu}, W_{-1}^{a} \boldsymbol{\nu}=2 L_{-1} t^{a} \boldsymbol{\nu}$, and

$$
\begin{aligned}
W_{-2}^{a} \boldsymbol{\nu} & =\left(\frac{3}{2} L_{-2}+L_{-1}^{2}\right) t^{a} \boldsymbol{\nu} \\
L_{-1}^{4} \boldsymbol{\nu} & =\left(5 L_{-2} L_{-1}^{2}-\frac{9}{4} L_{-2}^{2}-L_{-3} L_{-1}+\frac{3}{2} L_{-4}\right) \boldsymbol{\nu} .
\end{aligned}
$$

It follows that the special subspace is eight-dimensional, and that a basis can be chosen to be

$$
\mathcal{V}_{3 / 8}^{\mathrm{s}}=\left\langle\nu^{ \pm}, L_{-1} \nu^{ \pm}, L_{-1}^{2} \nu^{ \pm}, L_{-1}^{3} \nu^{ \pm}\right\rangle
$$

Any highest weight representations of the triplet algebra of conformal weight $h=-1 / 8,1$ or $3 / 8$ is automatically irreducible, and therefore isomorphic to $\mathcal{V}_{-1 / 8}, \mathcal{V}_{1}$ and $\mathcal{V}_{3 / 8}$, respectively. This follows from the fact that a reducible representation would have to contain a subrepresentation whose highest weight would be a descendant singular vector. However, the above analysis implies that such singular vectors do not exist.

On the other hand, there exist reducible highest weight representations with $h=0$. In particular, the highest weight representation $\mathcal{M}_{0}$, generated from a ground state $\omega$ of conformal weight $h=0$ and $j=0$, which does not satisfy (5), but only the constraints coming from (11) and (2) is reducible. It contains two subrepresentations of type $\mathcal{V}_{1}$, whose ground states are given as

$$
\begin{array}{ll}
\psi_{1}^{+}=W_{-1}^{+} \omega, & \psi_{2}^{+}=\left(W_{-1}^{0}+\frac{1}{2} L_{-1}\right) \omega, \\
\psi_{1}^{-}=\left(-W_{-1}^{0}+\frac{1}{2} L_{-1}\right) \omega, & \psi_{2}^{-}=W_{-1}^{-} \omega .
\end{array}
$$

3. Fusion and indecomposable representations. To calculate the fusion of these representations we use the same algorithm as in [14 to which we also refer for more details. Let us illustrate the method by explaining a simple case explicitly, the fusion of $\mathcal{V}_{-1 / 8} \times \mathcal{V}_{1}$. On general grounds, as was shown in [12], we know that the space of lowest energy states in the fusion product is contained in $\mathcal{V}_{-1 / 8}^{\mathrm{s}} \otimes \mathcal{V}_{1}^{0}$. However, this ten-dimensional space is too large since we have

$$
\begin{aligned}
\Delta\left(W_{-2}^{a}\right)(\mu \otimes \boldsymbol{\psi})-4 \Delta\left(L_{-2}\right)\left(\mu \otimes t^{a} \boldsymbol{\psi}\right)= & W_{-2}^{a}(\mu \otimes \boldsymbol{\psi})-4\left(L_{-1} \mu \otimes t^{a} \boldsymbol{\psi}\right) \\
& -\frac{1}{2}\left(\mu \otimes t^{a} \boldsymbol{\psi}\right), \\
\Delta\left(W_{-1}^{a}\right)(\mu \otimes \boldsymbol{\psi})-3 \Delta\left(L_{-1}\right)\left(\mu \otimes t^{a} \boldsymbol{\psi}\right)= & W_{-2}^{a}(\mu \otimes \boldsymbol{\psi})-3\left(L_{-1} \mu \otimes t^{a} \boldsymbol{\psi}\right) .
\end{aligned}
$$

To lowest level in the fusion product we can thus replace

$$
W_{-2}^{a} \mu \otimes \boldsymbol{\psi} \mapsto-\frac{3}{2} \mu \otimes t^{a} \boldsymbol{\psi}, \quad L_{-1} \mu \otimes \boldsymbol{\psi} \mapsto-\frac{1}{2} \mu \otimes \boldsymbol{\psi},
$$

and choose representatives $\mu \otimes \psi^{ \pm}$for the two-dimensional ground space $\left(\mathcal{V}_{-1 / 8} \otimes \mathcal{V}_{1}\right)_{\mathrm{f}}^{0}$. The action of $L_{0}$ and $W_{0}^{a}$ on this ground space is easily determined as

$$
\Delta\left(L_{0}\right)=\frac{3}{8} \mathbf{1}, \quad \Delta\left(W_{0}^{a}\right)=\frac{1}{2} t^{a}
$$


and the fusion product is thus a highest weight representation of type $h=3 / 8, j=1 / 2$. As was argued before, this representation is automatically irreducible, and therefore it is not necessary to analyse the states at higher level. We have thus shown that $\left.\right|^{2}$

$$
\left(\mathcal{V}_{-1 / 8} \otimes \mathcal{V}_{1}\right)_{\mathrm{f}}=\mathcal{V}_{3 / 8}
$$

In the same way we can show that the fusion with $\mathcal{V}_{0}$ is trivial, and that

$$
\left(\mathcal{V}_{3 / 8} \otimes \mathcal{V}_{1}\right)_{\mathrm{f}}=\mathcal{V}_{-1 / 8}
$$

The remaining fusion products involve potentially reducible representations. A calculation to lowest level is then not sufficient, and we analysed the product spaces up to level two. Before describing the results in more detail, let us comment briefly on the question whether the algorithm terminates. It is clear from $(6,0,8)$ that we can replace successively the $W$-modes by $L$-modes, and that the special subspace relations for the $L$-modes close among themselves. It is then clear, by the same argument as in [14, that the algorithm always stops.

In the remaining cases we found

$$
\begin{aligned}
\left(\mathcal{V}_{1} \otimes \mathcal{V}_{1}\right)_{\mathrm{f}} & =\mathcal{V}_{0}, \\
\left(\mathcal{V}_{-1 / 8} \otimes \mathcal{V}_{-1 / 8}\right)_{\mathrm{f}} & =\mathcal{R}_{0} \\
\left(\mathcal{V}_{-1 / 8} \otimes \mathcal{V}_{3 / 8}\right)_{\mathrm{f}} & =\mathcal{R}_{1}, \\
\left(\mathcal{V}_{3 / 8} \otimes \mathcal{V}_{3 / 8}\right)_{\mathrm{f}} & =\mathcal{R}_{0}
\end{aligned}
$$

where $\mathcal{R}_{0}, \mathcal{R}_{1}$ are generalised highest weight representations whose structure can (schematically) be described by the following diagram:
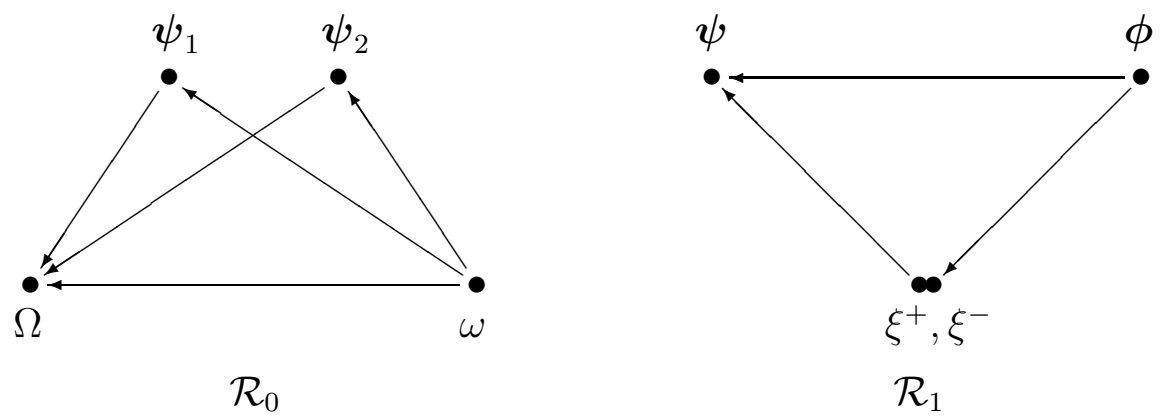

Here each vertex represents an irreducible representation, $\mathcal{V}_{0}$ in the bottom row and $\mathcal{V}_{1}$ in the top row. An arrow $A \longrightarrow B$ indicates that the vertex $B$ is in the image of $A$ under the action of the triplet algebra.

\footnotetext{
${ }^{2}$ We should mention that our calculations only imply upper bounds for the fusion rules. This is however sufficient to prove rationality.
} 
3a. The representation $\mathcal{R}_{0}$ : This representation is generated from a cyclic vector $\omega$ of $h=0$ forming a Jordan block with $\Omega$. It is an extension of $\mathcal{V}_{0}$ by $\mathcal{M}_{0}$. The defining relations are

$$
\begin{aligned}
L_{0} \omega & =\Omega, \\
W_{0}^{a} \omega & =0 .
\end{aligned}
$$

This is the only possible generalised highest weight representation which can be obtained from a two-dimensional Jordan block at $h=0$. It also follows from (4) that larger Jordan blocks at $h=0$ are incompatible with the vacuum null-relations.

3b. The representation $\mathcal{R}_{1}$ : This representation is generated from a doublet $\phi^{ \pm}$of cyclic states of weight $h=1$. It has two ground states $\xi^{ \pm}$at $h=0$ and another doublet $\psi^{ \pm}$at $h=1$ forming $L_{0}$ Jordan blocks with $\phi^{ \pm}$. The defining relations are

$$
\begin{aligned}
L_{0} \boldsymbol{\psi} & =\boldsymbol{\psi}, & W_{0}^{a} \boldsymbol{\psi} & =2 t^{a} \boldsymbol{\psi}, \\
L_{0} \boldsymbol{\xi} & =0, & W_{0}^{a} \boldsymbol{\xi} & =0, \\
L_{-1} \boldsymbol{\xi} & =\boldsymbol{\psi}, & W_{-1}^{a} \boldsymbol{\xi} & =t^{a} \boldsymbol{\psi}, \\
L_{1} \boldsymbol{\phi} & =-\boldsymbol{\xi}, & W_{1}^{a} \boldsymbol{\phi} & =-t^{a} \boldsymbol{\xi}, \\
L_{0} \boldsymbol{\phi} & =\boldsymbol{\phi}+\boldsymbol{\psi}, & W_{0}^{a} \boldsymbol{\phi} & =2 t^{a} \boldsymbol{\phi} .
\end{aligned}
$$

We stress that $\psi^{ \pm}$and $\phi^{ \pm}$form a Jordan block with respect to $L_{0}$ but that they remain uncoupled with respect to $W_{0}^{a}$. On higher levels there are also Jordan blocks for $W_{0}^{a}$. At first, it would seem that there might be inequivalent representations of this structure, as were obtained for the Virasoro case in [14] (see also [15]). However, in contrast to the situation for the Virasoro algebra, the vacuum representation of the triplet algebra contains null vectors, and the compatibility with these constraints restricts this freedom. It turns out, that there is no free parameter, and that the representation given above is the only one of its type.

The indecomposable representations $\mathcal{R}_{0}$ and $\mathcal{R}_{1}$ of the triplet algebra decompose (with respect to the Virasoro subalgebra) into the indecomposable representations $\mathcal{R}_{2 m-1,1}$ and $\mathcal{R}_{2 m, 1}$ of the Virasoro algebra. In particular, as a subrepresentation of $\mathcal{R}_{0}$ and $\mathcal{R}_{1}$, the characteristic parameters of these Virasoro representations are fixed. We have determined these parameters, and found that they agree in all cases $\left(\mathcal{R}_{m, 1}\right.$ with $\left.m \leq 5\right)$ with those determined in [14]. The representations of the Virasoro algebra appearing in fusion products at $c=-2$ are therefore precisely those which come from the allowed representations of the triplet algebra.

Finally, we calculated the fusion products involving the indecomposable representations. For the first four fusion products a calculation to lowest level was again sufficient; the other cases were calculated to level two.

$$
\left(\mathcal{V}_{-1 / 8} \otimes \mathcal{R}_{0}\right)_{\mathrm{f}}=2 \mathcal{V}_{-1 / 8} \oplus 2 \mathcal{V}_{3 / 8}
$$




$$
\begin{aligned}
\left(\mathcal{V}_{-1 / 8} \otimes \mathcal{R}_{1}\right)_{\mathrm{f}} & =2 \mathcal{V}_{-1 / 8} \oplus 2 \mathcal{V}_{3 / 8}, \\
\left(\mathcal{V}_{3 / 8} \otimes \mathcal{R}_{0}\right)_{\mathrm{f}} & =2 \mathcal{V}_{-1 / 8} \oplus 2 \mathcal{V}_{3 / 8}, \\
\left(\mathcal{V}_{3 / 8} \otimes \mathcal{R}_{1}\right)_{\mathrm{f}} & =2 \mathcal{V}_{-1 / 8} \oplus 2 \mathcal{V}_{3 / 8}, \\
\left(\mathcal{V}_{1} \otimes \mathcal{R}_{0}\right)_{\mathrm{f}} & =\mathcal{R}_{1}, \\
\left(\mathcal{V}_{1} \otimes \mathcal{R}_{1}\right)_{\mathrm{f}} & =\mathcal{R}_{0} \\
\left(\mathcal{R}_{0} \otimes \mathcal{R}_{0}\right)_{\mathrm{f}} & =2 \mathcal{R}_{0} \oplus 2 \mathcal{R}_{1}, \\
\left(\mathcal{R}_{0} \otimes \mathcal{R}_{1}\right)_{\mathrm{f}} & =2 \mathcal{R}_{0} \oplus 2 \mathcal{R}_{1}, \\
\left(\mathcal{R}_{1} \otimes \mathcal{R}_{1}\right)_{\mathrm{f}} & =2 \mathcal{R}_{0} \oplus 2 \mathcal{R}_{1} .
\end{aligned}
$$

It follows from these results that the set of representations $\mathcal{V}_{0}, \mathcal{V}_{1}, \mathcal{V}_{-1 / 8}, \mathcal{V}_{3 / 8}$ and $\mathcal{R}_{0}, \mathcal{R}_{1}$, is closed under fusion. The triplet algebra at $c=-2$ defines therefore a rational logarithmic conformal field theory.

It is natural to speculate that this conclusion will also hold for the other $(1, q)$ models. We have checked this explicitly for the next case, the triplet algebra at $c=-7$, and the results confirm this conjecture.

4. Characters and modular transformations. The characters of the irreducible representations of the triplet algebra have been calculated in [8] (see also [7]). From these, and the explicit description of the indecomposable representations, we can derive the characters of all the above representations. In more detail we have

$$
\begin{aligned}
\chi_{\mathcal{V}_{0}}(\tau) & =\frac{1}{2}\left(\eta(\tau)^{-1} \theta_{1,2}(\tau)+\eta(\tau)^{2}\right), \\
\chi_{\mathcal{V}_{1}}(\tau) & =\frac{1}{2}\left(\eta(\tau)^{-1} \theta_{1,2}(\tau)-\eta(\tau)^{2}\right), \\
\chi_{\mathcal{V}_{-1 / 8}}(\tau) & =\eta(\tau)^{-1} \theta_{0,2}(\tau), \\
\chi_{\mathcal{V}_{3 / 8}}(\tau) & =\eta(\tau)^{-1} \theta_{2,2}(\tau), \\
\chi_{\mathcal{R}_{0}}(\tau) & =2 \eta(\tau)^{-1} \theta_{1,2}(\tau), \\
\chi_{\mathcal{R}_{1}}(\tau) & =2 \eta(\tau)^{-1} \theta_{1,2}(\tau) .
\end{aligned}
$$

It turns out that the space generated by the last four characters is invariant under the action of the modular group, and that each has a suitable transformation property under $S$, whereas the $S$ transformation of $\chi_{\mathcal{V}_{0}}(\tau)$ and $\chi_{\mathcal{V}_{1}}(\tau)$ involves coefficients which are themselves functions of $\tau$, i.e.

$$
\begin{aligned}
& \chi_{\mathcal{V}_{0}}(-1 / \tau)=\frac{1}{4} \chi_{\mathcal{V}_{-1 / 8}}(\tau)-\frac{1}{4} \chi_{\mathcal{V}_{3 / 8}}(\tau)-\frac{i \tau}{2} \eta(\tau)^{2}, \\
& \chi_{\mathcal{V}_{1}}(-1 / \tau)=\frac{1}{4} \chi_{\mathcal{V}_{-1 / 8}}(\tau)-\frac{1}{4} \chi_{\mathcal{V}_{3 / 8}}(\tau)+\frac{i \tau}{2} \eta(\tau)^{2} .
\end{aligned}
$$


There have been attempts to give an interpretation of this transformation property in terms of generalised characters [7].

The representations $\mathcal{R}_{0}$ and $\mathcal{R}_{1}$ contain the irreducible subrepresentations $\mathcal{V}_{0}$ and $\mathcal{V}_{1}$, respectively, and the set of representations $\mathcal{V}_{-1 / 8}, \mathcal{V}_{3 / 8}$ and $\mathcal{R}_{0}, \mathcal{R}_{1}$ is already closed under fusion. This suggests that the fundamental building blocks of the theory are these four representations (c.f. also [15]). Two of its characters are the same, and so the definition of the modular matrices is ambiguous. It turns out that there is a one parameter freedom to define these matrices so that the relations of the modular group, $S^{4}=11$ and $(S T)^{3}=S^{2}$ are satisfied, [5 and a unique solution for which the charge conjugation matrix $S^{2}$ is a permutation matrix. In the basis of $\chi_{\mathcal{R}_{0}}, \chi_{\mathcal{R}_{1}}, \chi_{\mathcal{V}_{-1 / 8}}, \chi_{\mathcal{V}_{3 / 8}}$ this solution is given as

$$
S=\left(\begin{array}{cccc}
-\frac{1}{2} i & \frac{1}{2} i & \frac{1}{4} & -\frac{1}{4} \\
\frac{1}{2} i & -\frac{1}{2} i & \frac{1}{4} & -\frac{1}{4} \\
1 & 1 & \frac{1}{2} & \frac{1}{2} \\
-1 & -1 & \frac{1}{2} & \frac{1}{2}
\end{array}\right) \quad T=\left(\begin{array}{cccc}
e^{2 \pi i / 12} & 0 & 0 & 0 \\
0 & e^{2 \pi i / 12} & 0 & 0 \\
0 & 0 & e^{-\pi i / 12} & 0 \\
0 & 0 & 0 & e^{11 \pi i / 12}
\end{array}\right)
$$

It is intriguing that a formal application of Verlinde's formula leads to fusion rule coefficients which are positive integers. These do not reproduce the fusion rules we have calculated. This is not surprising, as, for example, this set of representations does not contain the vacuum representation, i.e. a representation which has trivial fusion rules. Even more drastically, the fusion matrix corresponding to the representation $\mathcal{V}_{-1 / 8}$ which, in the above basis, is given as

$$
N_{-1 / 8}=\left(\begin{array}{cccc}
0 & 0 & 1 & 0 \\
0 & 0 & 0 & 1 \\
2 & 2 & 0 & 0 \\
2 & 2 & 0 & 0
\end{array}\right)
$$

is not diagonalisable, and the same is true for the matrix corresponding to $\mathcal{V}_{3 / 8}$. However, a slight modification of Verlinde's observation still holds: the above $S$ matrix transforms the fusion matrices into block diagonal form, where the blocks correspond to the two one-dimensional and the one two-dimensional representation of the fusion algebra.

Acknowledgements. We would like to thank Wolfgang Eholzer, Michael Flohr and Gérard Watts for useful discussions.

M.R.G. is supported by a Research Fellowship of Jesus College, Cambridge, and H.G.K. by a Research Fellowship of Sidney Sussex College, Cambridge. This work has also been supported in part by PPARC.

The computer calculations were performed on computers purchased on EPSRC grant GR/J73322, using a MAPLE package written by H.G.K.

\footnotetext{
${ }^{3}$ All different choices lead to equivalent representations of the modular group.
} 


\section{References}

[1] L. Rozansky and H. Saleur, Quantum field theory for the multi-variable Alexander-Conway polynomial, Nucl. Phys. B376 (1992) 461, hep-th/9203069.

[2] V. Gurarie, Logarithmic operators in conformal field theory, Nucl. Phys. B410 (1993) 535, hep-th/9303160.

[3] A. Bilal and I. I. Kogan, On gravitational dressing of 2D field theories in chiral gauge, Nucl. Phys. B449 (1995) 569, hep-th/9503209.

[4] J.-S. Caux, I. I. Kogan and A. M. Tsvelik, Logarithmic Operators and Hidden Continuous Symmetry in Critical Disordered Models, Oxford University preprint OUTP-95-62 S, hepth/9511134, Nov. 1995.

[5] Z. Maassarani and D. Serban, Non-unitary conformal field theory and logarithmic operators for disordered systems, preprint SPHT-T96/037, hep-th/9605062, Apr. 1996.

[6] H. Saleur, Polymers and percolation in two dimensions and twisted $N=2$ supersymmetry, Nucl. Phys. B382 (1992) 486, hep-th/9111007.

[7] M. A. Flohr, On modular invariant partition functions of conformal field theories with logarithmic operators, preprint CSIC-IMAFF-42-1995, hep-th/9509166, Sept. 1995.

[8] H. G. Kausch, Curiosities at $c=-2$, preprint DAMTP 95-52, hep-th/9510149, Oct. 1995.

[9] G. M. Watts, A crossing probability for critical percolation in two dimensions, preprint DAMTP 96-19, cond-mat/9603167, Mar. 1996.

[10] I. I. Kogan and N. E. Mavromatos, World-Sheet Logarithmic Operators and Target Space Symmetries in String Theory, Oxford University preprint OUTP-95-50 P, hep-th/9512210, Dec. 1995.

[11] M. R. Gaberdiel, Fusion in conformal field theory as the tensor product of the symmetry algebra, Int. J. Mod. Phys. A9 (1994) 4619, hep-th/9307183.

[12] W. Nahm, Quasirational fusion products, Int. J. Mod. Phys. B8 (1994) 3693, hepth/9402039.

[13] M. Flohr, On Fusion Rules in Logarithmic Conformal Field Theories, preprint IASSNSHEP-96/54, hep-th/9605151, May 1996.

[14] M. R. Gaberdiel and H. G. Kausch, Indecomposable Fusion Products, preprint DAMTP 96-36, hep-th/9604026, Apr. 1996.

[15] F. Rohsiepe, Nichtunitäre Darstellungen der Virasoro-Algebra mit nichttrivialen Jordanblöcken, Diploma dissertation, Bonn University, 1996.

[16] H. G. Kausch, Extended conformal algebras generated by a multiplet of primary fields, Phys. Lett. B259 (1991) 448.

[17] W. Eholzer, A. Honecker and R. Hübel, How complete is the classification of $\mathcal{W}$-symmetries?, Phys. Lett. B308 (1993) 42.

[18] B. L. Feigin, T. Nakanishi and H. Ooguri, The Annihilating Ideals of Minimal Models, Int. J. Mod. Phys. A7 (1992) 217. 